\title{
ОБ УРАВНЕНИЯХ СТАЦИОНАРНОГО ГРАВИТАЦИОННОГО ПОЛЯ В ПОСТ-НЕРЕЛЯТИВИСТСКОМ ПРИБЛИЖЕНИИ
}

\section{A. KOPPEL. STATSIONAARSE GRAVITATSIOONIVALLJA VORRANDITEST POST-MITTERELATIVIST. LIKUS LAHENDUSES \\ A. KOPPEL. ON POST-NONRELATIVISTIC EQUATIONS OF THE STATIONARY GRAVITATIONAL FIELD}

В данной работе поставлена цель получить основные уравнения, определяющие стационарное гравитационное поле (СГ-поле) в пост-нерелятивистском приближении, и проанализировать их с учетом возможности существования неньютонова нерелятивистского предела $\left[{ }^{1}\right]$. При этом изучение уравнений движения материальных объектов в таком поле в наши задачи не входит.

1. Будем исходить * из уравнений $(1.10)-(1.14)$ работы $\left[{ }^{2}\right]$. В качестве тензора $T_{\mu v}$ принимаем тензор энергии-импульса идеальной жидкости. Тогда на основе формул $(2.9)-(2.11)$ работы $\left[{ }^{2}\right]$ величины $\stackrel{\vec{j}, \mathrm{~S}}{\mathrm{~S}}$ (1.14) получаются в виде

$$
\begin{aligned}
& \varrho=-4 \pi G \mu_{0}-\frac{4 \pi G}{c^{2}}\left[\mu_{0}\{\lambda+2 \hat{k}(\vec{u} \otimes \vec{u})\}+3 p_{0}\right]+O\left(\frac{1}{c^{4}}\right), \\
& \vec{j}=\frac{4 \pi G \mu_{0}}{c^{2}}[\overrightarrow{(1)}+2 \vec{u}]+O\left(\frac{1}{c^{4}}\right), \\
& \mathbf{S}=-\frac{4 \pi G \mu_{0}}{c^{2}} \stackrel{(0)}{\mathbf{k}}+O\left(\frac{1}{c^{4}}\right) .
\end{aligned}
$$

Здесь $\mu_{0}-$ плотность вещества, $p_{0}-$ давление, а $\vec{u}-$ скорость вещества в предельном эвклидовом 3 -пространстве $\stackrel{(0)}{V}$. Видим, что полученные разложения имеют члены только с четными степенями $1 / c$. Поэтому разложения для основных характеристик СГ-поля принимаем также в виде

$$
\lambda=1+\frac{\stackrel{(2)}{\lambda}}{c^{2}}+\frac{(4)}{c^{4}}+O\left(\frac{1}{c^{6}}\right)
$$

* В данной статье использованы обозначения и определения работы $\left[{ }^{2}\right]$. 


$$
\begin{aligned}
& \vec{\sigma}=\frac{\stackrel{(1)}{\vec{\sigma}}}{c}+\frac{(3)}{c^{3}}+O\left(\frac{1}{c^{5}}\right), \\
& \mathrm{k}=\stackrel{(0)}{\mathrm{k}}+\frac{\stackrel{(2)}{\mathrm{k}}}{c^{2}}+O\left(\frac{1}{c^{4}}\right) \text {. }
\end{aligned}
$$

Для дальнейших вычислений выведем две вспомогательные формулы. Пусть в пространстве $\bar{V}_{3}$ с метрическим тензором k существует некоторый вектор $\vec{A}$, причем

$$
\vec{A}=\frac{1}{c^{n}}\left[\stackrel{(n)}{\vec{A}}+\frac{1}{c^{2}} \stackrel{(n+2)}{\vec{A}}+O\left(\frac{1}{c^{4}}\right)\right]
$$

( $n$ - нуль или положительное целое число). Тогда для дивергенции и ротора этого вектора получим с учетом (6) следующие выражения:

$$
\begin{gathered}
\nabla \vec{A}=\frac{1}{c^{n}}\left[\stackrel{(0)}{\nabla} \vec{A}+\frac{1}{c^{2}}\left\{\stackrel{(0)}{\nabla} \stackrel{(n+2)}{\vec{A}}-\frac{1}{2}(\stackrel{(0)}{k})^{-1} \stackrel{(2)}{k} \stackrel{(0)}{\nabla} \vec{A}+\right.\right. \\
\left.\left.\left.+\frac{1}{2} \stackrel{(n)}{\nabla}[\stackrel{(0)}{(k)})^{-1} \stackrel{(2)}{k} \stackrel{(n)}{\vec{A}}\right]+\stackrel{(0)}{\nabla^{\prime}} \stackrel{(n)}{\vec{A}}\right\}+O\left(\frac{1}{c^{4}}\right)\right], \\
\nabla \times \vec{A}=\frac{1}{c^{n}}\left[\stackrel{(0)}{\nabla} \times \stackrel{(n)}{\vec{A}}+\frac{1}{c^{2}}\left\{\stackrel{(0)}{\nabla} \times \stackrel{(n+2)}{\vec{A}}-\frac{1}{2} \stackrel{(0)}{(k)}^{-1} \stackrel{(2)}{k} \stackrel{(0)}{\nabla} \times \stackrel{(n)}{\vec{A}}\right\}+O\left(\frac{1}{c^{4}}\right)\right] .
\end{gathered}
$$

Здесь $k \equiv \operatorname{det}\left\|k_{l m}\right\|=\stackrel{(0)}{k}+\frac{\stackrel{(2)}{k}}{c^{2}}+O\left(\frac{1}{c^{4}}\right), \stackrel{(0)}{\nabla}-\quad$ градиентный оператор в эвклидовом пространстве $\stackrel{(0)}{V}$ с с метрическим тензором $\stackrel{(0)}{\mathrm{k}}$, a

$$
\stackrel{(0)}{\nabla^{\prime}} \stackrel{(n)}{\vec{A}} \equiv(\stackrel{(0)}{k})^{-1 / 2}\left[\left(\stackrel{(0)}{(k)^{1 / 2}} \stackrel{(2)}{k}^{s p} \stackrel{(n)}{A}{ }_{p}\right], s,\right.
$$

где $\stackrel{(2)}{k^{s p}}=-\stackrel{(2)}{k_{l m}} \stackrel{(0)}{k^{s l} k^{p m}}$ и $\quad k_{l m}=\stackrel{(0)}{k_{l m}}+\frac{k_{l m}}{c^{2}}+O\left(\frac{1}{c^{4}}\right)$.

В силу (1)-(10) уравнения СГ-поля (формулы (1.10)-(1.13) работы $\left.\left[{ }^{2}\right]\right)$ принимают теперь вид

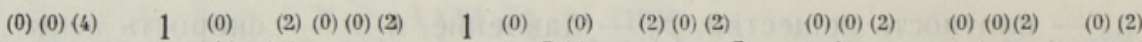
$\nabla \nabla \lambda=\frac{1}{2}(k)^{-1} k \nabla \nabla \lambda-\frac{1}{2} \nabla\left[(k)^{-1} k \nabla \lambda\right]-\nabla^{\prime} \nabla \lambda+\mathbf{k}(\nabla \lambda \otimes \nabla \lambda)+$

(2) (0) (0)

(0) (0) $\stackrel{(3)}{\rightarrow}$

$+2 \lambda \nabla \nabla\left(\psi^{2}\right)-4 \mathbf{k}(\nabla \psi \otimes \vec{\sigma})-4 \mathbf{k}(\nabla \psi \otimes \vec{\psi} \psi)+$

$$
+8 \pi G\left[2 \mu_{0} \mathbf{k}(\vec{u} \otimes \vec{u})+3 p_{0}\right],
$$

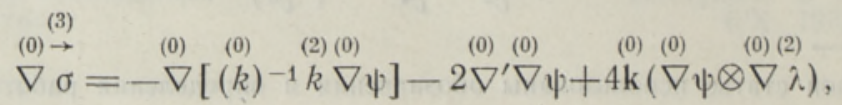




$$
\begin{aligned}
& \stackrel{(0)}{\nabla} \times \stackrel{(3)}{\vec{\sigma}}=-16 \pi G \mu_{0} \vec{u}, \\
& \stackrel{(2)}{\mathrm{P}}=-2(\stackrel{(0)}{\nabla} \psi \otimes \stackrel{(0)}{\nabla} \psi) .
\end{aligned}
$$

Здесь выписаны только члены, имеющие такой же порядок малости, как и первые релятивистские поправки величин $\varrho, \vec{j}$ и $\mathrm{S}$. В полученных уравнениях учтены как формула $\stackrel{\stackrel{(1)}{\rightarrow}}{\sigma}=2 \stackrel{(0)}{\nabla} \psi$, так и уравнения нерелятивистского СГ-поля (3.1)-(3.3) работы $\left[{ }^{2}\right]$.

2. Прежде всего отметим существенное следствие, вытекающее из (14). Видим, что в рассматриваемом приближении неньютонов предел еызывает искривление 3 -пространства $\bar{V}_{3}$ (конформное пространство по Фоку $\left.\left[{ }^{3}\right]\right)$. В случае ньютонова предела всегда можно выбрать координатную систему, где $\psi=0$, и, следовательно, получить $\stackrel{(2)}{\mathbf{P}}=0$, т. е. и в пост-нерелятивистском приближении $\bar{V}_{3}$ остается эвклидовым.

После определения $\stackrel{(2)}{\mathrm{k}}$ на основе (14) из уравнений (12) и (13) по

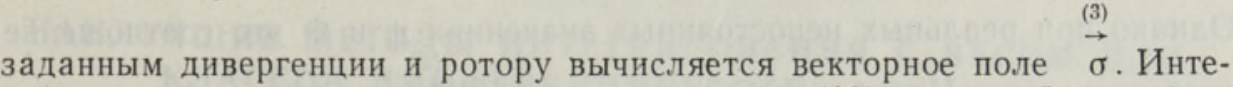
ресно отметить, что, хотя согласно формуле (13) вихревой характер этого поля определяется движением вещества, в общем случае $\vec{\sigma}$ не сугубо соленоидальный вектор, так как в силу неисчезающего нереляти(0) (3)

вистского вихревого потенциала $\nabla \vec{\sigma} \neq 0$. Вне областей, занимаемых источниками, $\mu_{0} \vec{u}=0$, и $\stackrel{(3)}{\vec{\sigma}}$ является градиентным. В этом случае первая релятивистская поправка к нерелятивистскому вихревому потенциалу определяется из уравнения Пуассона (12). Поправка $\stackrel{\text { (4) }}{\lambda}$ к величине $\stackrel{(2)}{\lambda}=2\left(\Phi-\psi^{2}\right) \quad(\Phi-$ ньютонов потенциал) вычисляется также из уравнения типа Пуассона (11).

3. Будем теперь рассматривать СГ-поле с ньютоновым пределом $(\psi=0)$. Тогда в силу (14) можно полагать $\stackrel{(2)}{\mathrm{k}}=0$, а уравнениям (11) - (13) придать соответственно вид

$$
\begin{aligned}
& \stackrel{(0)(0)(4)}{\nabla \nabla \lambda}=4 \mathrm{k}(\stackrel{(0)}{\nabla} \Phi \otimes \stackrel{(0)}{\nabla} \Phi)+8 \pi G\left[2 \mu_{0} \mathrm{k}(\vec{u} \otimes \vec{u})+3 p_{0}\right], \\
& \stackrel{(0)}{\nabla \sigma}=0, \\
& \stackrel{(0)}{\nabla} \times \stackrel{(3)}{\vec{\sigma}}=-16 \pi G \mu_{0} \vec{u} .
\end{aligned}
$$

Видим, что уравнения (16) и (17) для $\stackrel{(3)}{\vec{\sigma}}$ формально совпадают с уравнениями квазистационарного магнитного поля при наличии источников поля. Для сравнения отметим, что градиентный нерелятивистский вектор $\stackrel{\stackrel{(1)}{\sigma}}{\vec{\sigma}}$ (см. формулу (3.5) работы $\left[{ }^{2}\right]$ ) тоже удовлетворяет уравнениям типа (16) и (17), но с полностью исчезающей правой частью. 
Сказанное порождает проблему: можно ли рассматривать неньютонов нерелятивистский предел как некоторый «упрощенный вариант» (с уравнениями без источников для вихревого поля) пост-ньютонова приближения СГ-поля, данного уравнениями (15)-(17)?

Чтобы положительно ответить на поставленный вопрос, мы должны,

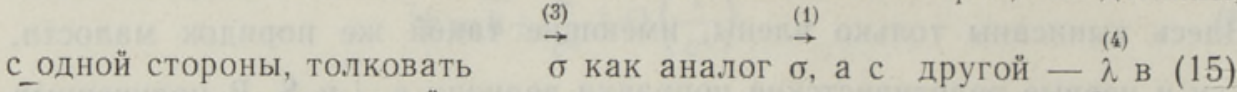
ка̄к аналог определенной поправки к ньютонову потенциалу Ф, имеющей место в неньютоновом пределе. Для областей вне источников

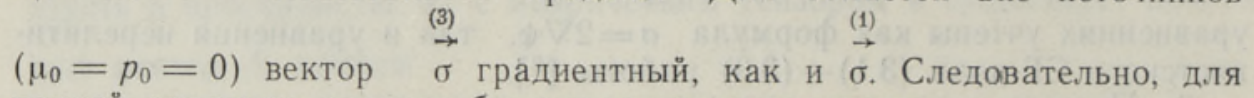
полной аналогии должны бы выполняться равенства

$$
\stackrel{(3)}{\vec{\sigma}}=2 \stackrel{(0)}{\nabla} \psi, \quad \stackrel{(4)}{\lambda}=-2(\psi)^{2} .
$$

Но тогда из (15) мы получили бы

$$
\stackrel{(0)}{\mathrm{k}}(\stackrel{(0)}{\nabla} \psi \otimes \stackrel{(0)}{\nabla} \psi)+\stackrel{(0)}{\mathbf{k}}(\stackrel{(0)}{\nabla} \Phi \otimes \stackrel{(0)}{\nabla} \Phi)=0 .
$$

Однако при реальных непостоянных значениях $\psi$ и Ф это соотношение (4) несостоятельно. Итак, невозможно толковать $\lambda$ как аналог поправки к ньютонову потенциалу $\Phi$ в неньютоновом пределе, т. е. в случае СГ-полей трактовать соотношения для неньютонова предела как просто некие «остатки» соотношений для пост-ньютонова приближения - нельзя. Неньютонов предел релятивистских гравитационных полей, на существование которого впервые указывалось в работе $\left[{ }^{1}\right]$, имеет, по-видимому, самостоятельное значение и специфическую сущность.

Отметим еще, что в силу уравнения ньютоновой теории гравитации $\stackrel{(0)}{\nabla} \nabla \Phi=4 \pi G \mu_{0} \quad$ уравнению (15) можно придать также вид

$$
\stackrel{(0)(0)}{\nabla} \stackrel{(2)}{\Phi}=4 \pi G\left\{2 \mu_{0}[\stackrel{(0)}{\mathrm{k}}(\vec{u} \otimes \vec{u})-\Phi]+3 p_{0}\right\},
$$

где

$$
\stackrel{(2)}{\Phi}=\frac{\stackrel{(4)}{\lambda}}{2}-(\Phi)^{2}
$$

Сравнение этого результата с формулой (3.4) работы $\left[{ }^{2}\right]$ показывает, что наличие вихревого потенциала $\psi$ в выражении для характерного скаляра $\lambda$ СГ-поля добавляет в случае неньютонова нерелятивистского предела к решению уравнения Пуассона или Лапласа $1+\frac{2 \Phi}{c^{2}}$ отрицательный член $-\frac{2\left(\psi^{2}\right)}{c^{2}}$, в то время как в случае поля с ньютоновым пределом в пост-ньютоновом приближении к решению уравнения Пуассона или Лапласа $1+\frac{2 \Phi}{c^{2}}+\frac{2 \Phi}{c^{4}}$ прибавляется положительный член $+\frac{2(\Phi)^{2}}{c^{4}}$. Следует иметь в виду, что скаляр $\lambda$ (точнее его квадратный корень) определяет по существу энергию пробной частицы в данном СГ-поле (см. также $\left.\left[{ }^{4}\right]\right)$.

В заключение автор выражает благодарность Х. Кересу за внимание к работе и обсуждение результатов. 


\title{
Л И ТЕРА Т У РА
}

1. Керес Х., ЖЭТФ, 48, 1319 (1965).

2. К оппель А., Изв. АН ЭССР, Физ. Матем., 24, 297 (1975).

3. Фок В. А., Теория пространства, времени и тяготения, М., 1961, с. 272.

4. Л андау Л. Д., Л ифшиц Е. М., Теория поля, М., 1973, с. 320.

Тартуский государственный университет
Поступила в редакцию 8/VII 1975

\author{
EESTI NSV TEADUSTE AKADEEMIA TOIMETISED. 25. KOIDE \\ FOOSIKA * MATEMAATIKA. 1976, NR. 2
}

ИЗВЕСТИЯ АКАДЕМИИ НАУК ЭСТОНСКОП ССР. ТОМ 25 ФИЗИКА * МАТЕМАТИКА, 1976, № 2

\section{Б. БОЯНОВ}

\section{НАИЛУЧШИЕ МЕТОДЫ ИНТЕГРИРОВАНИЯ С ВЕСОМ ДЛЯ КЛАССОВ ДИФФЕРЕНЦИРУЕМЫХ ФУНКЦИЙ}

B. BOJANOV. PARIMAD KAALUGA INTEGREERIMISMEETODID DIFERENTSEERUVATE FUNKTSIOONIDE KLASSIDELE

B. BOYANOV. BEST METHODS OF WEIGHTED INTEGRATION FOR CLASSES OF DIFFERENTIABLE FUNCTIONS

Пусть $W_{q}^{(r)}(M ; a, b), 1 \leqslant q \leqslant \infty, r=1,2 \ldots-$ класс всех функций, заданных на конечном интервале $[a, b]$, имеющих $(r-1)$-ю абсолютно непрерывную производную и $r$-ю, удовлетворяющую неравенству $\left\|f^{(r)}\right\|_{q} \leqslant M$. Пусть функция $\omega(x)$ суммируема и положительна на $[a, b]$. Рассмотрим задачу о построении наилучшего (т. е. с минимальной оценкой погрешности) метода приближения интеграла $I(f)=\int_{a}^{b} \omega(x) f(x) d x$ на классе $W_{q}^{(r)}(M ; a, b)$ с помощью информации $\left\{f^{(k)}\left(x_{i}\right)\right\}_{k=0}^{r-1}$ в $n$ фиксированных точках $\left\{x_{i}\right\}_{1}^{n}: a \leqslant x_{1}<\ldots<x_{n} \leqslant b$. Поскольку по лемме С. А. Смоляка [ $\left.{ }^{1}\right]$ (см. также $\left[{ }^{2}\right]$ ) среди наилучших методов есть линейный, то ограничимся только методами вида

$$
I(f) \approx \sum_{i=1}^{n} \sum_{k=0}^{r-1} A_{i k} f^{(k)}\left(x_{i}\right)
$$

Обсзначим через $\pi_{m}$ множество всех многочленов степени не выше $m$. Как показал М. И. Левин $\left[{ }^{3}\right]$, наилучший метод является точным для многочленов класса $\pi_{r-1}$.

Для построения наилучшего метода будем использовать следующую простую идею: сначала построим элементарные наилучшие формулы вида (1) по информации сперва в одном, а затем в обоих концах интервала интегрирования, а потом покажем, что метод, основанный на вычислении каждого из интегралов $\int_{a}^{x_{1}}, \int_{x_{1}}^{x_{2}}, \ldots, \int_{x_{n}}^{b}$ элементарным методом (т. н. составной), будет наилучшим. 